Mens

revue d'histoire intellectuelle de l'Amérique française

\title{
Roy McMurtry, les droits des Franco-Ontariens et la nation canadienne
}

\section{Linda Cardinal et Stéphane Lang}

Volume 7, numéro 2, printemps 2007

URI : https://id.erudit.org/iderudit/1024126ar

DOI : https://doi.org/10.7202/1024126ar

Aller au sommaire du numéro

Éditeur(s)

Centre de recherche en civilisation canadienne-française

ISSN

1492-8647 (imprimé)

1927-9299 (numérique)

Découvrir la revue

Citer cet article

Cardinal, L. \& Lang, S. (2007). Roy McMurtry, les droits des Franco-Ontariens et la nation canadienne. Mens, 7(2), 279-311. https://doi.org/10.7202/1024126ar

\section{Résumé de l'article}

Les travaux sur les idées constitutionnelles au Canada pendant les années 1970 et 1980 négligent trop souvent la pluralité idéologique existant parmi les principaux acteurs de l'époque. Cet article tente de pallier cette lacune en analysant l'apport de l'ancien procureur général de l'Ontario, Roy McMurtry, à la redéfinition de l'identité nationale canadienne dans le contexte des bouleversements constitutionnels de 1982. Le texte montre que le nom de McMurtry devrait aussi être associé aux débats constitutionnels du tournant des années 1980 au même titre que celui de Pierre Trudeau. Il porte aussi sur son attitude à l'égard de la minorité franco-ontarienne en raison du rôle clé qu'il souhaitait qu'elle joue dans le rapprochement entre le Québec et le reste du pays. Associé au courant red tory, McMurtry a ainsi mis son héritage intellectuel au service des droits des minorités. 


\title{
ROY MCMURTRY, LES DROITS DES FRANCO-ONTARIENS ET LA NATION CANADIENNE
}

\author{
Linda Cardinal et Stéphane Lang \\ Chaire de recherche sur la francophonie \\ et les politiques publiques \\ Université d'Ottawa
}

\section{Résumé}

Les travaux sur les idées constitutionnelles au Canada pendant les années 1970 et 1980 négligent trop souvent la pluralité idéologique existant parmi les principaux acteurs de l'époque. Cet article tente de pallier cette lacune en analysant l'apport de l'ancien procureur général de l'Ontario, Roy McMurtry, à la redéfinition de l'identité nationale canadienne dans le contexte des bouleversements constitutionnels de 1982. Le texte montre que le nom de McMurtry devrait aussi être associé aux débats constitutionnels du tournant des années 1980 au même titre que celui de Pierre Trudeau. Il porte aussi sur son attitude à l'égard de la minorité franco-ontarienne en raison du rôle clé qu'il souhaitait qu'elle joue dans le rapprochement entre le Québec et le reste du pays. Associé au courant red tory, McMurtry a ainsi mis son héritage intellectuel au service des droits des minorités.

\section{Abstract}

Scholarly research on Canadian constitutional thought in the 1970s and 1980s often neglects the ideological pluralism that characterised the era's leading figures. The present article seeks to mitigate this oversight by examining the contribution 
of former Ontario attorney general Roy McMurtry to the redefinition of Canadian identity during the constitutional upheaval of 1982. The authors argue that McMurtry was no less significant to the constitutional debate of the early 1980s than was Pierre Trudeau. The article also examines McMurtry's attitude towards Ontario's French-speaking minority and the role that he hoped it would play in bridging the gap between Quebec and the rest of Canada. A Red Tory, McMurtry mustered his intellectual beritage in the service of minority rights.

Roy McMurtry est procureur général de l'Ontario de 1975 à 1985. Il est ensuite nommé Haut-Commissaire du Canada en Grande-Bretagne, poste qu'il occupe jusqu'en 1988. En 1991, il devient juge en chef adjoint de la Cour supérieure de l'Ontario, Division de première instance et juge en chef de cette même Cour en 1994. Depuis 1996, il est juge en chef de l'Ontario. À 75 ans, l'on peut dire qu'il a consacré toute sa carrière au service public.

Notre intérêt pour l'œuvre de McMurtry est lié à son rôle, à titre de procureur général de l'Ontario, dans les débats constitutionnels des années 1980 et, de façon concomitante, dans le développement des services en français au sein du système judiciaire de la province. Certes, il n'a pas innové lorsqu'il a choisi de mouler la position constitutionnelle de l'Ontario sur le projet du gouvernement fédéral de rapatrier unilatéralement la Constitution ${ }^{2}$. Toutefois, il a formulé un discours sur la nation canadienne qui diffère substantiellement de celui de Pierre Trudeau. En outre, si pour Trudeau, à en juger par ses déclarations à l'époque du Lac Meech, les droits linguistiques des minorités servent à protéger l'individu contre tous droits collectifs ${ }^{3}$, pour McMurtry, ils doivent plutôt s'insérer dans le cadre de droits accordés aux communautés linguistiques, ce qui l'incitera à appuyer la reconnaissance du statut de société distincte du Québec. Ses idées n’ont 
toutefois pas retenu l'attention des historiens ni celle des commentateurs de la période ${ }^{4}$. Pourtant, nous aurions intérêt à mieux étudier la façon dont il se représentait la nation et les droits des minorités afin d'en approfondir la signification dans le contexte du débat sur l'identité canadienne qui s'élabore à l'époque. Les travaux font habituellement rimer l'évolution du Canada avec la pensée constitutionnelle de Trudeau. Pensons, pour nous en convaincre, aux études de la question réalisées par Kenneth McRoberts ou Guy Laforest ${ }^{5}$. Les commentateurs n'ont pas tort de procéder à un tel rapprochement mais leur discours sert aussi à gommer l'existence d'une pluralité idéologique bien réelle au sein de l'historiographie et de la politique canadiennes ${ }^{6}$. Ce texte tentera de pallier cette lacune en analysant l'apport de McMurtry à la redéfinition de l'identité nationale au Canada dans le contexte des bouleversements constitutionnels de 1982. Il portera aussi sur son attitude à l'égard de la minorité franco-ontarienne en raison du rôle clé qu'il souhaitera qu'elle joue dans le rapprochement entre le Québec et le reste du pays. En effet, il a contribué à forger une représentation particulière de la nation canadienne au cœur de laquelle se trouve un engagement qui peut surprendre à l'égard des droits des Franco-Ontariens et des minorités francophones hors Québec de façon plus générale. Associé au courant red tory, il a mis son héritage intellectuel au service des droits des minorités ${ }^{7}$.

Ce texte servira donc à montrer que le nom de McMurtry devrait être associé aux débats constitutionnels des années 1980 au même titre que l'ont été ceux de Pierre Trudeau ou de Jean Chrétien ${ }^{8}$. Au centre de notre réflexion, nous retrouvons notamment son interprétation de la Charte canadienne des droits et libertés comme un document fondateur de la nation canadienne. Pour certains, il est ironique que le Québec rejette un document du type de la Charte, d'autant plus 
que celle-ci est tributaire d'un raisonnement civiliste étranger au monde de la common law ${ }^{9}$. Or, ce texte montrera que l'esprit de McMurtry plane aussi sur le document de l'époque.

Par ailleurs, si l'on connaît mal son apport au constitutionnalisme canadien, son rôle clé dans le développement des services en français en Ontario a été reconnu par l'ensemble du milieu francophone de la province, que l'on pense au doctorat honorifique que lui a conféré l'Université d'Ottawa en 1983 ou encore aux nombreux discours qu'il a prononcés devant les membres de l'Association des juristes d'expression française de l'Ontario (AJEFO). Il a fait preuve, très tôt dans sa carrière de politicien, d'un engagement indéfectible envers la cause du bilinguisme, des services en français et du respect de la citoyenneté des Franco-Ontariens.

Ce texte est divisé en trois parties. Dans un premier temps, nous présentons quelques éléments du parcours de McMurtry. Nous étudierons ainsi sa pensée comme une forme d'intervention dans l'histoire du Canada et non uniquement comme un témoignage sur une période révolue. Dans un deuxième temps, nous présenterons sa conception des minorités et de la nation canadienne. Dans un troisième temps, nous montrerons comment il a fait valoir ses idées constitutionnelles dans la tourmente constitutionnelle de 1982. En somme, nous verrons comment il est devenu un acteur important sur la scène politique canadienne.

\section{Roy McMurtry, le réformateur}

Originaire de Toronto, McMurtry se définit lui-même comme étant d'origine irlandaise, venant d'une famille protestante et orangiste par surcroit ${ }^{10}$. Il a fréquenté les meilleurs écoles du Canada anglais, pensons aux collèges d'Upper Canada et de St. Andrew's. En 1954, il reçoit un baccalauréat ès Arts de l'Université de Toronto et en 1958, un diplôme en 
droit de Osgoode Hall. Pendant ses années à l'université, il rencontre et se lie d'amitié avec William Davis, le futur premier ministre de l'Ontario.

McMurtry a pratiqué le droit dans la ville reine pendant dix-sept ans. Il s'est fait connaître, notamment, par sa prise de position en 1970 contre l'entrée en vigueur de la Loi sur les mesures de guerre ${ }^{11}$. En 1975, le premier ministre conservateur Davis lui demande de se présenter en politique. Il espère que McMurtry réussira à réformer le système judiciaire de la province. À cette époque, la société ontarienne est très divisée sur les réformes sociales, en particulier sur la question de la réforme du droit de la famille. Entre 1971 et 1975, quatre procureurs généraux se sont cassé les dents en proposant des solutions de compromis ${ }^{12}$. Les conservateurs traditionalistes ne voulaient rien entendre des réformes touchant le rôle des hommes et des femmes au sein de la famille. En particulier, la Commission de réforme du droit de l'Ontario (Ontario Law Reform Commission) propose de reconnaitre les conjoints de fait (common law relationships) ainsi que la prise en compte de la contribution des mères au foyer et dans l'éducation des enfants dans les procédures de divorce. La Commission remettait ainsi en cause le principe de propriété sur les biens acquis pendant le mariage donnant le droit aux femmes divorcées d'accéder aux biens pour lesquels les titres de propriété revenaient au mari. Devant ces deux mesures, les conservateurs traditionalistes s'inquiètent de la possibilité de voir une hausse substantielle du taux de divorce et une augmentation du nombre de familles constituées hors de l'institution du mariage. Or, une partie croissante de l'électorat estime au contraire que le gouvernement doit se faire le promoteur d'une nouvelle réalité sociale et implanter les réformes au système judiciaire de la Commission de réforme du droit de l'Ontario. Les groupes féministes revendiquent une protection juridique pour 
un meilleur accès au travail et la possibilité de se lancer en affaires en gardant pour elles des droits exclusifs sur les biens acquis $^{13}$. Bref, la famille et le travail des femmes sont au centre de débats sociaux importants et les conservateurs se trouvent divisés sur la question.

Malgré ces difficultés, Davis remporte les élections de 1975 mais forme un gouvernement minoritaire, l'électorat s'étant déplacé à gauche vers le NPD et les Libéraux ${ }^{14}$. La même année, il nomme McMurtry procureur général et lui demande de concevoir une réforme jugée nécessaire pour assurer la stabilité du gouvernement. Il relèvera avec succès le défi lancé par le premier ministre. Entre 1975 et 1984, il fait adopter une cinquantaine de lois provinciales servant à transformer l'appareil judiciaire ontarien. Il innove en mettant en place un premier réseau de cliniques juridiques communautaires $^{15}$. Dans le domaine du droit privé, il présente en 1978 le Family Law Reform Act, qui comporte un compromis dans le cas de divorce limitant les biens divisibles à ceux de la famille et excluant les biens commerciaux. Profitant d'un partenariat avec les avocats du droit privé et la Commission de réforme du droit de l'Ontario, il réforme aussi le droit des successions et introduit la Loi sur le privilège dans l'industrie de la construction. En 2004, McMurtry explique que ses réformes et innovations ont ainsi contribué à réduire le coût des poursuites au civil et le temps d'attente dans les procès au criminel ${ }^{16}$.

La réforme du système judiciaire est jumelée à d'autres initiatives visant à le rendre bilingue. Il s'agit d'ailleurs de l'une des rares occasions où les droits des francophones se trouvent au centre des préoccupations d'un législateur au moment clé d'une réforme majeure touchant la structure administrative d'un ministère. Or, le dossier des services en français n'est pas une priorité pour McMurtry au moment où il est nommé procureur général de l'Ontario. À l'époque, la struc- 
ture gouvernementale des services en français est restreinte. Depuis la Commission Laurendeau-Dunton, le premier ministre de l'Ontario, John Robarts, a mis en place quelques mesures afin de mieux servir la population francophone de sa province $^{17}$. Ainsi, en 1965, il crée un comité de coordination des services en français, l'Advisory Committee on French Language Services. Il permet l'utilisation du français à l'Assemblée législative de la province. En 1967, il annonce la mise sur pied d'écoles secondaires publiques de langue française $^{18}$. En 1971, devant la proposition du gouvernement fédéral d'adopter la Charte de Victoria, l'Ontario accepte le principe de rendre ses lois disponibles en français. La même année, le 3 mai, le nouveau premier ministre conservateur, Bill Davis, réitère, devant l'Assemblée législative de l'Ontario, l'engagement de la province à offrir des services en anglais et en français là où le nombre le justifie et là où cela est possible. «Mr. Speaker, dit-il, it is clear that Ontario has made a solid commitment to the principle of bilingualism as a matter of equity for our residents and as a large contribution to the continued and future strength of Canada ${ }^{19}$. " Ainsi, le gouvernement de la province publie une première politique relative à la prestation de services en français en Ontario. Celle-ci comprend l'obligation de traduire les documents destinés au public et de répondre en français à toute demande écrite de renseignements. Elle crée aussi les premières régions désignées. Enfin, en 1974, un Conseil consultatif des affaires francoontariennes est créé.

En somme, lorsque McMurtry se joint au gouvernement ontarien en 1975, la structure des services en français se limite à quelques initiatives de la part du premier ministre Robarts et à la reconnaissance du principe des services en français par le gouvernement Davis. Dans le domaine judiciaire, l'arrivée de McMurtry a aussi pour effet de changer 
favorablement les choses pour les Franco-Ontariens ${ }^{20}$. En effet, le dossier des services en français devient rapidement sa priorité car dès les premières semaines de son mandat, il constate que la communauté franco-ontarienne se mobilise afin de réclamer l'accès à une justice en français. Jacqueline Pelletier, une enseignante au collège Algonquin à l'époque, vient de refuser de payer une contravention rédigée en anglais et veut être jetée en prison. Elle suivait l'exemple de Raymond Desrochers, animateur social et candidat du NPD dans Prescott-Russell, qui avait refusé de renouveler l'immatriculation de sa voiture devant l'absence de services en français au gouvernement ontarien ${ }^{21}$. Qui plus est, pendant les semaines qui suivent, des dizaines de Franco-Ontariens vont se présenter devant les cours de justice en demandant d'être " menottés » pour les mêmes raisons que Jacqueline Pelletier.

De plus, au moment même où McMurtry fait son entrée en politique, les francophones de l'Ontario œuvrent à redéfinir plus largement la justice comme une question de justice sociale. À l'aide d'une campagne médiatique pancanadienne, le mouvement C'est l'temps! voit le jour. Il veut que le premier ministre Davis tienne sa promesse du 3 mai 1971 par laquelle il s'était engagé à fournir des services gouvernementaux bilingues aux citoyennes et citoyens de l'Ontario. De façon plus précise, le mouvement revendique une véritable politique de bilinguisme dans les services gouvernementaux, le «droit fondamental des Franco-Ontariens de s'exprimer librement - c'est-à-dire sans traducteur interposé - devant les tribunaux de leur province » et des codes civil et criminel de l'Ontario accessibles en français, « comme ils le sont d'ailleurs au Québec et au Nouveau-Brunswick ${ }^{22} »$. Il est appuyé par l'Association canadienne-française de l'Ontario (ACFO), l'Association des enseignantes et des enseignants 
francophones de l'Ontario (AEEFO) et la Fédération des femmes canadiennes-françaises (FFCF ${ }^{23}$.

Devant ce mouvement, qu'allaient dire les Québécois du traitement réservé aux Franco-Ontariens ? McMurtry était souvent préoccupé par l'opinion du Québec et de l'image que l'Ontario projetait des francophones dans sa province «sœur ${ }^{24}$ ». Pour sa part, le mouvement C'est l'temps! pouvait se féliciter d'avoir réussi à créer un rapport de force favorable à une plus grande prise en compte des préoccupations des Franco-Ontariens par le gouvernement de la province. La première initiative de McMurtry a été la création, au mois de juin 1976, d'un projet-pilote de tribunal bilingue à Sudbury. Malgré un succès mitigé auprès de la population francophone de cette région, l'expérience s'étend en 1977 à Ottawa, puis dans les comtés de Prescott et Russell, dans l'Est ontarien où vit une majorité de francophones. McMurtry met alors sur pied un Comité consultatif de langue française qui comprend des juristes anglophones et francophones de l'Ontario ainsi que des juristes francophones du Québec. Il souhaite apprendre de la longue expérience du bilinguisme judiciaire pratiqué au Québec. C'est sur les conseils de ce Comité consultatif qu'il choisit de privilégier "une approche "étape par étape" » afin de «naviguer avec succès dans les eaux turbulentes ${ }^{25}$ » de sa province. Décriée par l'ACFO à l'époque, la stratégie vise d'abord à développer la capacité bilingue du système judiciaire et à augmenter le nombre de juristes bilingues par la création d'un programme de formation. L'approche séduit d'autant plus McMurtry que l'accent est mis sur le développement de nouvelles ressources humaines et ce, sans trop bousculer la majorité anglophone de la province.

Entre 1977 et 1984, plusieurs initiatives sont directement attribuables aux travaux de ce Comité. En outre, ses membres définissent les modalités institutionnelles, adminis- 
tratives et législatives qui orienteront le développement des services en français dans le secteur de la justice. En 1978, le gouvernement ontarien modifie la Loi sur les jurys et la Loi sur l'organisation judiciaire. La même année, McMurtry appuie la création d'un programme de common law en français à l'Université d'Ottawa. En 1979, Étienne Saint-Aubin, un avocat francophone impliqué dans le projet-pilote de Sudbury, devient le premier coordonnateur des services en français au sein du ministère du Procureur général. Ces événements constituent également un contexte favorable à la mobilisation d'un nouveau réseau d'acteurs, celui des avocats francophones. En effet, en 1980, l'Association des juristes d'expression française de l'Ontario (AJEFO) voit le jour. Avalisé par McMurtry, le nouveau groupe social se lance dans l'activisme judiciaire. À titre d'exemple, en établissant le Centre de traduction et de documentation juridique en partenariat avec l'Université d'Ottawa, l'AJEFO veut contribuer, de concert avec le bureau du coordonnateur des services en français du ministère du Procureur général, à l'élaboration des articles 135 et 136 de la Loi de 1984 sur les tribunaux judiciaires qui ont fait de l'anglais et du français les langues officielles des tribunaux ontariens, à la rédaction de la Loi de 1986 sur les services en français et à la traduction des Lois refondues de l'Ontario de 1990. Ces lois-cadres serviront de base à l'extension de l'usage du français devant tous les tribunaux ontariens.

McMurtry est sûrement conscient qu'il vient de cautionner une nouvelle force politique qui exercera une certaine influence sur la communauté francophone et qui pourra tenter de l'orienter politiquement. Il n'hésite pas à reconnaître l'AJEFO d'autant plus que le nouveau regroupement accepte les anglophones bilingues dans ses rangs.

La première revendication politique de la nouvelle association, formulée lors de son congrès fondateur, est d'exi- 
ger que l'Ontario devienne officiellement bilingue. De plus, comme nous le verrons plus loin, ses membres ne se gêneront pas pour critiquer l'approche étapiste de McMurtry, notamment lors des négociations constitutionnelles en 1981 et 1982. Mais retenons surtout de cette période que l'AJEFO parvient à s'insérer dans une structure de gouvernance établie par le procureur général et son coordonnateur des services en français. Le Comité consultatif (jusqu'en 1984) et l'Université d'Ottawa, par l'intermédiaire de son programme de common law en français et le Centre de traduction et de documentation juridique (CTDJ), font également partie de cette structure. Nous sommes ainsi en présence d'un nouveau réseau d'acteurs publics qui permet, pour la première fois, de débattre de la situation des services en français dans le domaine de la justice en Ontario. Par ailleurs, même si le mouvement C'est l'temps! et les autres porte-parole de la communauté francophone ne sont pas intégrés directement à l'élaboration de la politique ontarienne dans le domaine de la justice, ils continuent d'influencer le cours des choses par le moyen de leur participation au débat politique plus large sur l'avenir de la fédération canadienne.

De façon concomitante, le succès de McMurtry dans la réforme du système judiciaire ontarien et les rapports de confiance qu'il a établis avec les juristes francophones contribuent à rehausser son prestige auprès de Davis. Il a fait ses preuves. Il a maintenu la stabilité de la province et permis au gouvernement conservateur de garder le pouvoir. Il ne sera donc pas étonnant de le voir jouer un rôle de premier plan dans le domaine constitutionnel et la rédaction de la Charte. En effet, de 1976 à 1980, McMurtry devient le conseiller de Davis en matière constitutionnelle. Au lendemain de l'élection du gouvernement de Trudeau en 1980 et à la veille de la campagne référendaire du Québec, il est un des principaux 
acteurs pour l'Ontario dans les négociations constitutionnelles qui s'amorcent. Au mois d'avril 1981, il plaide pour l'Ontario devant la Cour suprême du Canada en faveur d'un rapatriement unilatéral de la Constitution par le gouvernement fédéral. À l'été de 1981, il parcourt le pays pour convaincre les provinces anglophones d'adopter la position de l'Ontario. Au mois de novembre 1981, il conclut une entente avec Jean Chrétien et Roy Romanow qui pose les bases du rapatriement de la Constitution. Il contribue également à l'inscription, dans la Charte, du droit à l'éducation des minorités de langue officielle ${ }^{26}$.

\section{Roy McMurtry, les droits des minorités et la nation canadienne}

Il est utile de revoir les éléments de la pensée de McMurtry sur les Franco-Ontariens et la conception des rapports entre les peuples et la nation qu'il échafaude dans le cadre des négociations constitutionnelles de 1980 et 1981. Ceux-ci servent à mieux faire apparaître sa contribution au projet d'édification de la nation canadienne et sa position favorable à la reconnaissance des droits des minorités.

Le 6 mars 1980, soit deux semaines après l'élection de Pierre Trudeau et trois mois avant le référendum québécois sur la souveraineté-association, McMurtry s'adresse aux membres du prestigieux Empire Club of Canada. Fréquenté par les milieux d'affaires et les dirigeants politiques ontariens, cette tribune a, par le passé, accueilli des personnalités politiques importantes dont les premiers ministres du Canada Louis StLaurent (1953) et Pierre Trudeau (1973), et de nombreux premiers ministres provinciaux. Des personnalités internationales y ont également été entendues, dont Winston Churchill, Indira Gandhi et Richard Nixon. Enfin, entre 1977 et 1981, l'Empire Club of Canada reçoit de nombreux acteurs impli- 
qués directement dans les débats sur la souveraineté du Québec et sur la Constitution canadienne. En 1977, le ministre péquiste des Finances et du Revenu, Jacques Parizeau, y prononce une conférence intitulée « Four months later». En 1979, le premier ministre ontarien Davis y parle du rôle de l'Ontario dans la Confédération. En janvier 1980, le premier ministre du Québec, René Lévesque, y présente, dans une conférence intitulée "On a working hypothesis », son projet de souveraineté-association et de question référendaire. En février 1980, le premier ministre du Canada, le conservateur Joe Clark, expose son programme, "The Challenge of Change » devant les membres du Club. En janvier 1981, le leader de l'opposition libérale du Québec, Claude Ryan, présente à ces derniers ses «Reflections on the Mood of Quebec Today ${ }^{27}$ ». Pour sa part, en 1980 également, McMurtry, dans une intervention majeure sur la nation au Canada, y révèle les fondements politiques de sa pensée constitutionnelle. Se référant à Ernest Renan, il compare la nation canadienne à une âme, le résultat combiné d'une histoire de gloires partagées et du désir de vivre ensemble ${ }^{28}$. Ce sont, selon lui, deux conditions nécessaires à l'existence d'une nation. C'est ce qu'il nomme le tissu national (national fabric) d'un pays. Au Canada, il considère que ce tissu est composé de deux fibres, la fibre française et la fibre anglaise - qu'il ne définit pourtant pas. Plus tard la même année, il dira toutefois que le sort du tissu national dépendra de la capacité de l'Ontario à améliorer la fibre linguistique et culturelle française de l'Ontario : «the extent to which we are able to strengthen the French linguistic and cultural fabric in Ontario will determine the extent to which we strengthen the fabric of our nation ${ }^{29}$ ».

McMurtry adhère au schéma de l'évolution du statut constitutionnel du Canada souhaité par les nationalistes canadiens-anglais depuis la Deuxième Guerre mondiale. Celui- 
ci comprend notamment le projet de rapatriement de la Constitution et celui de la création de la Charte des droits et libertés de 1982. Cela se voit clairement dans le récit qu'il fait des événements de l'époque. Dans un article de 1983 qu'il publie dans le Queen's Law Journal, McMurtry rappelle les grands moments de l'évolution constitutionnelle du Canada depuis la Confédération et souligne le caractère historique des événements de 1981 et 1982 pour la création d'une véritable nation canadienne. D'ailleurs, pour lui, le rapatriement de la Constitution " appeared to be long overdue for a sovereign and mature nation ${ }^{30}$ ". Il fait aussi part de son sentiment d'humiliation lorsqu'il parle de son voyage en Grande-Bretagne en compagnie de Jean Chrétien dans la première moitié de l'année 1981. Il est notamment choqué d'entendre les politiciens du Parlement de Londres débattre du statut constitutionnel du Canada dans la Chambre des communes dans des termes révélant " some worrisome paternalistic dimensions ». Dès lors, il est convaincu que le Canada doit immédiatement mettre un terme à son évolution coloniale.

En somme, McMurtry s'inscrit dans un mouvement de pensée au Canada anglais qui souhaite voir naître une véritable nation canadienne. Il veut repenser la place des "fibres » anglaise et française qui, pour lui, constituent l'épicentre de ce tissu national canadien. Cette ambition explique d'ailleurs sa référence au Canada comme un " [un] pays, [une] nation, [une] alliance historique des Français et des Anglais voué à l'indépendance et qui a forgé la Confédération ${ }^{31} »$. Elle permet aussi de mieux comprendre le fait qu'il conçoive, dans son discours du 6 mars 1980, que ce tissu soit menacé, d'une part, par la présence d'un gouvernement souverainiste à Québec qui remet en cause l'existence même de la fédération canadienne et, d'autre part, par la montée de sentiments hostiles envers Ottawa, Toronto et Montréal en Alberta et à Terre- 
Neuve. Selon McMurtry, cette situation oblige l'Ontario à jouer un rôle particulier dans un processus de réconciliation nationale. Il considère aussi que les relations entre le Québec et l'Ontario seront déterminantes pour l'avenir du Canada. Selon lui, l'Ontario et le Québec sont des provinces sœurs de par leurs relations historiques remontant à la création du Haut et du Bas-Canada. Il ne donne pas de détails sur l'utilisation de son expression "provinces-sœurs ». Or, McMurtry est convaincu que les relations entre les deux provinces sont cruciales pour l'avenir du nouveau partenariat canadien ${ }^{32}$.

Dans cette vision, les Franco-Ontariens, par l'entremise de leurs juristes, seront appelés à jouer le beau rôle de promoteurs de la dualité nationale dans leur province. McMurtry n'en parle pas dans le discours qu'il prononce devant les membres de l'Empire Club of Canada. C'est plutôt en 1981, lors d'une rencontre de l'AJEFO, qu'il précise sa pensée sur les minorités. Dans son discours devant les juristes francoontariens, il se fait non seulement le défenseur de la dualité nationale, il les invite à s'affirmer comme citoyens. Il déclare :

Laissez-moi vous répéter que mon engagement à réserver ce rôle au français dans notre système judiciaire ontarien procède d'une conviction profonde que la dualité de notre nation est l'un des fondements de son existence. En votre qualité de citoyens francophones ontariens, vous avez le droit de participer pleinement à la vie de votre propre province tout en conservant votre identité linguistique. Il est donc tout à fait normal que vous comptiez sur votre gouvernement provincial pour soutenir ces aspirations légitimes ${ }^{33}$.

Ainsi, l'on peut penser que les Franco-Ontariens constituent la «fibre » française du tissu canadien de l'Ontario. Cette représentation de McMurtry se retrouvera d'ailleurs au cœur de la conception de la dualité nationale véhiculée par le 
gouvernement ontarien à l'époque. C'est aussi à cette même conception de la dualité qu'il se référera, dans la foulée des événements de 1982, dans ses discours sur la Charte. Pour lui, celle-ci est non seulement le reflet des traditions légales anglaise et française. Elle sert aussi à édifier le Canada en tant que nation et à protéger, du même coup, les droits des minorités :

The Charter represented a balance between the dominant English and French legal traditions as well as reflecting the plurality of our country as a whole. Above all, it represented what Canada stood for as a nation: a basic respect for individual rights subject only to the wise restraint that could be "demonstrably justified in a free and democratic society ${ }^{34}$.

Selon ce point de vue, qui accorde un statut de primauté aux cultures dont la tradition légale est reconnue comme un des fondements de la nation, les Canadiens d'expression française vivant en Ontario ne devraient plus se voir comme des minoritaires. Or, à la grande surprise de la communauté franco-ontarienne, l'Ontario refuse de souscrire à l'article 133 de l'Acte de l'Amérique du Nord britannique et de se déclarer bilingue. Les juristes francophones sont particulièrement déçus car dans les faits, le statut de minoritaire continue de s'appliquer aux francophones hors Québec. En 1983, le président de l'AJEFO va plus loin et déclare que non seulement l'Ontario n'est pas bilingue, il ne le deviendra pas car la province est et demeurera viscéralement " provinciale »; « [l]'Acte de 1867 et celui de 1982 n'ont rien changé à cet état de fait ; de facto et de jure, sauf en matière d'enseignement public depuis la maternelle jusqu'à la $13^{\mathrm{e}}$ année ». Selon l'AJEFO, l'Ontario souscrit aux thèses suivantes: "You don't legislate culture » et "Bilingualism if necessary, but not necessarily bilingualism ${ }^{35} »$. Néanmoins, en 1984, l'Ontario accorde la 
gestion scolaire partielle à sa minorité francophone $e^{36}$. De plus, la province modifie sa Loi sur les tribunaux judiciaires afin de rendre son système de justice officiellement bilingue.

McMurtry a été, par moments, surpris de constater que les Franco-Ontariens n'adhéraient pas toujours à sa conception des choses, ce qui ne l'a pas empêché d'approfondir sa conception de la dualité nationale et linguistique et d'y reconnaitre un rôle spécial au Québec dans le développement de la francophonie. Selon lui,

[1]e Québec est évidemment une région très spéciale du Canada, une province à laquelle tous les Francophones sont attachés en raison de son caractère unique. Vous suggérer qu'on peut vivre de la même manière à Toronto qu'on le peut dans la ville de Québec ne serait évidemment pas réaliste malgré les progrès importants [accomplis par le gouvernement de l'Ontario $]^{37}$.

Il croit aussi que la vitalité de la francophonie canadienne hors Québec dépend d'abord de l'apport social et culturel du Québec ${ }^{38}$. Pour cette raison, il estime désirable que le gouvernement ontarien obtienne l'appui moral du gouvernement québécois dans ses efforts pour permettre aux FrancoOntariens de s'épanouir. Mais celui-ci n'est pas indispensable à court terme. McMurtry estime, au demeurant, que cet appui ne pourra jamais venir d'un gouvernement péquiste. En novembre 1982, il déclare:

René Lévesque m'a dit, un jour, qu'il ne portait guère d'intérêt aux initiatives vis-à-vis du français et du bilinguisme hors Québec. Pour lui, la langue française et sa culture ne peuvent fleurir ou même survivre que dans la province de Québec. C'est de toute évidence à travers les efforts de particuliers comme vous [les juristes franco-ontariens] que son pessimisme ne sera pas inscrit dans l'histoire de notre pays ${ }^{39}$. 
Bref, au lendemain de l'accord constitutionnel de 1982, McMurtry estime plus que jamais nécessaire de promouvoir les relations entre les francophones du Québec et de l'Ontario. De ces relations entre francophones dépendra la perception des Québécois selon laquelle le Canada anglophone est réellement engagé à faire de la dualité linguistique un fondement de la nation canadienne.

\section{Roy McMurtry dans la tourmente constitutionnelle}

Le rôle de McMurtry dans les négociations constitutionnelles est méconnu et pourtant crucial. Avec la victoire du camp du Non au référendum de mai 1980, Trudeau annonce, au mois de septembre de la même année, qu'il va procéder unilatéralement au rapatriement de la Constitution, c'est-àdire sans le consentement des provinces. Le NouveauBrunswick et l'Ontario se rallient au gouvernement fédéral et McMurtry se présente alors comme le porte-étendard de la nation canadienne.

Le Québec et sept autres provinces traînent le gouvernement fédéral devant les tribunaux. En avril 1981, McMurtry présente la position de l'Ontario devant la Cour suprême du Canada. Adoptant une approche brutalement légaliste, il soutient que depuis 1867, c'est le gouvernement fédéral seul qui a négocié les amendements constitutionnels avec le gouvernement britannique. Les provinces n'ayant jamais participé à ces négociations, elles n'ont donc obtenu aucun pouvoir dans les mécanismes qui se sont développés par la suite ${ }^{40}$. L'approche inédite et controversée du procureur l'amène toutefois à décrire la situation comme l'expression d'une " anomalie historique » que seuls les efforts du fédéral pourront corriger. Pour l'Ontario, le rapatriement de la Constitution exigera en effet une nouvelle entente entre les provinces et le gouvernement fédéral et permettra de revoir la répartition des pou- 
voirs. Or, le Québec et les sept autres provinces soutiennent que cette analyse de la situation place le gouvernement fédéral dans une position d'autorité absolue par rapport à eux. Un juge teste aussi le réalisme de McMurtry au point de le mettre dans l'embarras en lui demandant ce que l'Ontario ferait dans l'éventualité où le gouvernement fédéral lui imposerait le bilinguisme officiel. Il répond simplement qu'une telle initiative serait « unwise politically ${ }^{41} »$. Et dire que l'Ontario se présente comme une province phare auprès des autres provinces anglophones en ce qui a trait au respect des droits des minorités.

La déclaration de McMurtry provoque des remous au sein de la communauté francophone. Non seulement penset-il que l'adoption d'une politique de bilinguisme officiel serait peu prudente en Ontario, il va plus loin et soutient que l'approche "étapiste » de l'Ontario est même meilleure que celle du Nouveau-Brunswick qui, en adoptant, en 1981, la Loi reconnaissant l'égalité des deux communautés linguistiques, a reconnu l'égalité entre les communautés anglophone et francophone de la province. Il réagit alors à une déclaration du premier ministre du Nouveau-Brunswick, Richard Hatfield, qui, dans le cadre d'une conférence de presse à $\mathrm{New}$ York, a expliqué au premier ministre de l'Ontario qu'il aurait "délibérément induit en erreur» les Québécois au moment du référendum en se faisant l'avocat d'un fédéralisme renouvelé comportant des droits accrus pour les francophones sans avoir vraiment l'intention de les octroyer. En tant que porteparole de l'Ontario, McMurtry critique vivement Hatfield qui, selon lui, fait « de grandes déclarations de principes quitte à combler plus tard et dans un avenir plus ou moins vague, la faille qui existe entre la promesse et son exécution ${ }^{42} »$. Il soutient que l'Ontario évite les déclarations fracassantes en procédant par étapes. Selon lui, la méthode ontarienne permet 
une meilleure compréhension des difficultés et de la réalité. Il s'agit donc d'« établir fermement et efficacement l'usage de la langue française, en créant, tout d'abord, la capacité de fournir de tels services en langue française, et ensuite seulement, en en garantissant la disponibilité par une $\operatorname{loi}^{43} »$. Selon McMurtry, la méthode étapiste de l'Ontario a l'avantage de ne pas créer trop d'attentes chez les francophones. Il existe toujours un fossé entre les lois et la réalité et ce n'est pas ce qu'il souhaite car il considère qu'un tel écart provoque ou engendre " cynisme et amertume ${ }^{44}$ ».

Or, la communauté franco-ontarienne ne croit pas que l'Ontario puisse donner des leçons au Nouveau-Brunswick ou au Québec. Déjà, en novembre 1980, l'ACFO soulignait dans une lettre adressée à McMurtry qu'il ne devait pas donner l'impression au Québec que les Franco-Ontariens étaient aussi bien traités que les Anglo-Québécois. Voici ce qu'on y lit :

Au cours de l'été 1980, vous avez été l'un des deux ministres ontariens associés à la négociation constitutionnelle. Cette responsabilité vous met en situation privilégiée pour expliquer aux Québécois la position constitutionnelle de l'Ontario. À moins d'amendements adéquats, l'adoption du présent projet de «Loi constitutionnelle de 1980 » perpétuerait la situation de « deux poids, deux mesures » entre les Franco-Ontariens et les Anglo-Québécois ${ }^{45}$.

La lettre souligne le refus de l'Ontario d'accorder la gestion scolaire aux Franco-Ontariens et de déclarer la province bilingue en vertu de l'article 133 de l'Acte de l'Amérique du Nord britannique. L'ACFO demande aussi à McMurtry de préciser concrètement les prochaines étapes à suivre en vue de rendre le système de justice ontarien complètement bilingue. Elle souhaite qu'il donne les échéanciers fixés par 
son ministère afin de permettre, par exemple, la rédaction de testaments et le dépôt d'actes de vente et de documents légaux en français.

Dans sa réponse ultérieure à l'ACFO, il continue toutefois de soutenir que "l'étapisme» offre «l'avantage de promouvoir une meilleure compréhension des services en français au sein de la population ontarienne ${ }^{46} »$. Il reprend le même argument lorsqu'il s'adresse aux membres de l'AJEFO quelques semaines plus tard en ajoutant que « cette compréhension est d'une grande importance car les constitutions sont des plus vivantes lorsqu'elles s'inspirent d'une volonté populaire ${ }^{47} »$.

McMurtry semble tenir deux discours sur le rôle du Québec et de l'Ontario envers leur minorité. Les deux provinces, d'après le procureur général, doivent respecter leur minorité linguistique, mais seul le Québec aurait à être bilingue car l'Ontario n'aurait pas les moyens de répondre aux besoins des Franco-Ontariens. Selon lui, " [c]'est en tant que province fondamentalement unilingue, dotée d'une très petite population francophone, que l'Ontario s'est établi, s'est joint à la Confédération et s'est développé. » Et McMurtry d'ajouter : "[l]e Québec, de son côté, s'est développé davantage en tant que province bilingue, comprenant une communauté anglophone relativement forte. » Selon lui,

[1] bilinguisme en Ontario n'a pas de racine historique ni de tradition, comme c'est le cas au Québec; cela explique sans doute pourquoi, au Québec, presque tous les juges, procureurs de la Couronne et avocats du Barreau, dans leur ensemble, sont bilingues, alors que très peu le sont en Ontario. Malgré la fermeté de notre engagement, nous n'avons pas le pouvoir de changer, d'un jour à l'autre, ces limitations créées par 200 ans d'histoire ${ }^{48}$. 
Par ailleurs, c'est au nom de l'intérêt supérieur du pays que l'Ontario veut appuyer la démarche de Trudeau. Ce faisant, l'Ontario va aller à l'encontre des propositions de Robarts qui, dans le cadre de la Commission sur l'unité canadienne en 1979, souhaitait redéfinir la Confédération en tenant davantage compte des préoccupations des provinces ${ }^{49}$. Ce changement d'attitude s'explique par le fait que le gouvernement Davis ne reconnait pas que le gouvernement péquiste puisse légitimement négocier une entente constitutionnelle avec le reste du Canada. Dès 1976, il considère que le point de vue des dirigeants péquistes n'est pas tout à fait au diapason des citoyens ordinaires. Il croit fermement que la création d'une constitution vraiment canadienne serait en soi un symbole qui obtiendrait l'appui des Québécois. En novembre 1981, McMurtry déclare, bien sincèrement, que « we do not believe we have isolated the province of Quebec, perhaps only the Governement of Quebec, and I hope the people of Quebec will see it that way ${ }^{50} \%$.

Entre 1980 et 1981, l'Ontario considère également que le gouvernement fédéral avait obtenu un mandat clair des citoyens du Québec pour trouver une solution constitutionnelle. Le gouvernement fédéral parlera donc au nom du peuple québécois. C'est dans ce même esprit que McMurtry explique qu'il n'y a pas eu de "nuit des longs couteaux ", c'est-à-dire de connivence entre les premiers ministres provinciaux afin d'isoler le Québec durant la nuit précédant les négociations finales de la nouvelle Constitution canadienne. En fait, pour lui, Lévesque était le principal responsable de la division entre le Québec et les autres provinces. Il considère que lorsque Lévesque et Trudeau se sont défiés pour soumettre le rapatriement et la Charte à un référendum, les provinces anglophones se seraient, elles aussi, senties trahies ${ }^{51}$. 
L'Ontario veut alors jouer un rôle déterminant pour arriver à un compromis. Au congrès de l'AJEFO, McMurtry exprime sa déception de constater qu'au Québec, on ne prête aucune attention au fait que les neuf autres provinces ont accepté que les droits à l'enseignement en français soient incorporés à la Constitution. Cela le choque de voir que le Québec ne reconnait pas l'esprit de bonne entente qui anime les autres provinces. Il estime que les signataires n'ont pas isolé le Québec et croit que le peuple québécois n'est pas d'accord avec l'attitude du gouvernement de Lévesque à l'égard de la Charte. Il se dit qu'au moins les Québécois devaient être satisfaits qu'aucun futur amendement constitutionnel ne portera atteinte à la dualité de la nation ${ }^{52}$.

De plus, pour montrer la bonne volonté de l'Ontario, il fait une déclaration historique devant le parlement de l'Ontario deux semaines après l'Accord constitutionnel de novembre 1981. Il annonce l'intention du gouvernement de la province de rendre son système judiciaire officiellement bilingue. Deux jours plus tard, il exprime au deuxième congrès de l'AJEFO sa fierté d'avoir réussi à rendre possible la tenue de procès bilingues ou en français à Toronto ${ }^{53}$. Mais ce geste ne reçoit pratiquement aucune attention au Québec. L'année suivante, il demande aux juristes francophones de faire connaître le vrai visage de l'Ontario aux citoyens du Québec car il estime que les médias perpétuent le sentiment de trahison et l'isolement du Québec: "The inaccurate perception of Ontario as firmly set in the past on the matter of French language rights can only foster this isolation. And so I call upon you once again to convey in your own contacts with the citizens of Quebec that the negative headlines do not tell the true story ${ }^{54}$.»

En 1989, McMurtry intervient dans le débat sur l'Accord du Lac Meech. Celui qui a été haut-commissaire du Ca- 
nada au Royaume-Uni de 1985 à 1988, soutient que l'Accord permettra de corriger l'erreur de 1982 d'avoir exclu le Québec de la « famille canadienne ». Il déclare, par ailleurs, que la clause de la société distincte n'aura pas une très grande portée réelle et estime que le Canada anglais doit l'appuyer non pas parce que les droits des anglophones seront protégés mais bien parce que le Québec a renoncé à sa demande d'un droit de veto particulier. Pour lui, c'était un compromis extraordinaire qui montrait un esprit de bonne volonté de la part du Québec ${ }^{55}$.

Néanmoins, McMurtry s'est toujours montré fier d'avoir joué un rôle déterminant dans la création de la Charte, document qu'il considère, en 2003, comme « un triomphe politique de compromis et d'accommodements » qui garantit aux futures générations le pouvoir de "renforcer le tissu de la nationalité " ("strengthen the fabric of nationhood $\left.{ }^{56} »\right)$. Or, si, au plan pancanadien, il adopte une approche légaliste pour prendre la défense des droits des minorités, au plan provincial, il insiste sur la nécessité d'adopter une approche " étapiste", pour ne pas brusquer les choses et ne pas créer de fausses attentes. Par ailleurs, McMurtry est loin d'être un francophobe et s'est fait publiquement le défenseur du fait français en Ontario à plusieurs reprises. En effet, il n'hésite pas à dénoncer la francophobie partout où il la rencontre. Ainsi, en 1983, il dénonce publiquement les éditoriaux francophobes du North Bay Nugget. Sa lettre mérite d'être soulignée car elle révèle le type de société qu'il souhaite que l'Ontario devienne. Il écrit, à l'époque, qu'il a été témoin toute sa vie durant de discrimination subtile ou carrément outrageuse contre les minorités non anglophones. Or, ajoute t-il, l'Ontario est pluraliste. Il est constitué d'une grande variété d'ethnies et de races et le gouvernement fera tout pour que les divers groupes linguistiques et culturels reconnaissent la dualité « of 
our nation ${ }^{57} \%$. Il n'en demeure pas moins que la vision idéale de la nation de McMurtry se raccroche à une réalité qui explique le caractère pragmatique de son " étapisme ». En effet, son projet de rendre le système juridique bilingue devait être "vendu » à un électorat composé en partie d'éléments francophobes. Qui plus est, dès 1976, la plupart des membres du cabinet, abstraction faite du premier ministre Davis lui-même, sont vivement aux efforts qu'il faits pour rendre le système judiciaire bilingue ${ }^{58}$. L'une des craintes de McMurtry était aussi de voir les membres de la profession juridique se polariser. Son propre comité consultatif sur les services en français était inquiet de la possibilité que les avocats anglophones perçoivent les réformes comme une obligation d'embaucher du personnel bilingue ou de devenir eux-mêmes bilingues. Les directives du 23 juin 1980 démontrent que cette crainte s'étend en fait à tous les intervenants anglophones du système judiciaire. On y explique que tous les efforts que déploie le procureur général afin de favoriser le bilinguisme du système judiciaire ontarien ne mettront aucun poste existant en jeu: "May I also reiterate, écrit McMurtry, that these policies should not be cause for concern among our staff since this will definitely not result in the employment of present staff being put in jeopardy ${ }^{59}$. $~ \mathrm{La}$ crainte d'un ressac des anglophones explique pourquoi McMurtry a repris les paroles du premier ministre John A. Macdonald devant les juristes francophones pour affirmer que «nous ne voulons pas nous situer aux limites extrêmes de nos droits. Nous sommes prêts à donner et à recevoir. Nous avons les moyens d'être justes et généreux, car nous sommes forts ${ }^{60}$. $" \mathrm{Ce}$ " nous » signifiait la majorité anglophone de l'Ontario. Les Franco-Ontariens ne pouvaient toujours pas tenir pour acquis le principe de l'égalité des deux peuples fondateurs au sein de leur province. 
Malgré les contradictions apparentes entre la théorie et la pratique des droits des minorités en Ontario, les idées mises de l'avant par McMurtry font partie des éléments clés du néonationalisme canadien contemporain. Elles constituent une des bases du nation-building canadien depuis les années 1980 . Or, jusqu'à présent, cette approche a été davantage associée à Trudeau qu'à McMurtry. Pourtant, ce dernier a aussi fortement contribué à élaborer une conception de la nation canadienne qui inscrit la langue comme une de ses dimensions fondamentales. Il souhaitait la construction d'un État-nation canadien qui desservirait les minorités francophones en leur aménageant un espace de droits au sein d'une nationalité dualiste. En effet, plus que Chrétien ou Romanow avec qui il a participé à l'édification du Canada de 1982, il aura réussi à insuffler à la nation canadienne un concept de dualité qui rappelle l'idée de partenariat entre deux peuples fondateurs.

En Ontario, avant McMurtry, Robarts avait aussi joué un rôle de premier plan en vue du renouvellement de la fédération mais selon une autre perspective. Principal architecte d'une politique de la main tendue vers le Québec qui revendique, à l'époque, un meilleur statut au sein de la fédération canadienne, il s'inspire de la thèse selon laquelle le Canada est constitué à la fois de provinces et de deux peuples fondateurs. Faut-il aussi le rappeler, en 1979, Robarts a coprésidé la Commission sur l'unité canadienne (Commission PépinRobarts) et signé un rapport en vue du renouvellement de la fédération, Se retrouver : observations et recommandations ${ }^{61}$. Ce document est le dernier à proposer une réforme de la fédération dans le sens d'une plus grande reconnaissance des provinces et des régionalismes au Canada. Or, Davis est un premier ministre qui reconnaît à Ottawa un plus grand rôle de fiduciaire de la nation canadienne comparativement à Robarts. 
Pour sa part, McMurtry ne partage pas la vision de ce dernier qui est plus ouvert à l'idée de réciprocité entre les gouvernements fédéral et provinciaux. Il est plus près de la position de Trudeau, qu'a épousée Davis qui ne veut pas faire trop de vagues tout en assurant le leadership dont on s'attend de l'Ontario dans le domaine de l'unité nationale. Toutefois, la nation de McMurtry n'est pas que juridique. Elle est aussi dualiste et culturelle. Nous lui devons notamment d'avoir tenté de donner un rôle spécial aux Franco-Ontariens dans cette conception même si ces derniers sont toujours en attente d'un politicien qui osera enfin déclarer la province officiellement bilingue.

\section{NOTES}

${ }^{1}$ Ce texte a été rendu possible grâce à un financement du bureau du Coordonnateur des services en français au ministère du Procureur général de l'Ontario pour réaliser un état des lieux des services en français dans le domaine de.la justice en Ontario. Nathalie Plante, Anik Sauvé et Chantal Terrien ont aussi collaboré à cette recherche. Nous les en remercions, ainsi que Marcel Castonguay, Anne-Andrée Denault et les évaluateurs anonymes de la revue, de leurs commentaires et suggestions. Ce texte a aussi fait l'objet d'une présentation au Centre de recherche en civilisation canadienne-française de l'Université d'Ottawa au mois de janvier 2006.

${ }^{2}$ Pour une chronologie des événements de l'époque, voir l'ouvrage de JeanFrançois Cardin et Claude Couture, Le Canada. Espace et différences, Québec, Presses de l'Université Laval, 1995.

${ }^{3}$ Voir Donald Johnston, dir., Lac Meech : Trudeau parle.., Montréal, Hurtubise HMH, 1989.

${ }^{4}$ La contribution de Roy McMurtry au dénouement des négociations constitutionnelles en 1982, tout comme celle de Roy Romanow, est parfois évoquée par les commentateurs, mais sous la forme de l'anecdote de l'Accord de la "cuisine " (Kitchen Accord). L'histoire sert à rappeler que les procureurs de l'Ontario et de la Saskatchewan McMurtry et Romanow et le ministre de la Justice du Canada, Jean Chrétien, ont jeté sur papier les compromis nécessai- 
res à une entente au terme d'une soirée festive. Voir Michel Vastel, Trudeau, le Québécois, Montréal, Éditions de l'Homme, 1989.

${ }^{5}$ Kenneth McRoberts, Misconceiving Canada. The Struggle for National Unity, Toronto, Oxford University Press, 1997 ; Guy Laforest, La fin du rêve canadien, Québec, Septentrion, 1992.

${ }^{6}$ Pour plus de détails, voir Jean-François Cardin et Claude Couture, op. cit.

${ }^{7}$ Les médias anglophones ontariens ont abondamment qualifié McMurtry de red tory pendant la course à la direction du Parti conservateur ontarien de 1985 qu'il a perdue aux mains de Frank Miller, associé à l'élément tory davantage à la droite du parti. La période de reconstruction du parti entre 1987 et 1990 a finalement mené au choix du néoconservateur Mike Harris comme chef du parti. Pour leur part, Roy McMurtry et William Davis étaient vus comme les seuls qui auraient pu rétablir les red tories en position de force. Voir Andrew Phillips, « Miller: From rags to riches to premier: Ontario's top Tory believes in capitalism, small-town values ", The Gazette, 28 janvier 1985, p. B1 ; Rosemary Speirs, "The need for reforming political party financing", Toronto Star, 30 mars 1987, p. A15; Harvey Schachter, «Tories need good policy, good strategy, and good luck to make comeback », The Whig-Standard, 15 septembre 1987, p.1 ; Ian Urquhart, «Flaherty's justice is not justice for all », Toronto Star, 10 janvier 2001, p. A21.

${ }^{8}$ La liste des ouvrages sur l'époque du rapatriement de la Constitution et l'adoption de la Charte canadienne est longue. Parmi les plus importants, mentionnons celui de Kenneth McRoberts, Un pays à refaire : l'échec des politiques constitutionnelles canadiennes, Montréal, Boréal, 1999.

${ }^{9}$ Graham Fraser, Sorry, I don't speak. French: confronting the Canadian crisis that won't go away, Toronto, McClelland \& Stewart, 2006.

${ }^{10}$ [Roy McMurtry, « Discours de l'honorable Roy R. McMurtry (Congrès de l'Association des juristes d'expression française de l'Ontario [dorénavant AJEFO]), novembre 1981, p. 1], Université d'Ottawa [dorénavant UO], Centre de recherche en civilisation canadienne-française [dorénavant $\mathrm{CRCCF}$ ], Fonds AJEFO (C126), C126-2/15/15.

"John A. MacNaughton, « Présentation de l'honorable Roy McMurtry, Procureur général de l'Ontario », Empire Club of Canada Addresses, vol. 77 (19791980), p. 280-282.

${ }^{12}$ Allan Lawrence (1971-1972), Dalton Bales (1972-1974), Robert Stanley Welch (1974-1975) et John Clement (1975). 
${ }^{13}$ H. Allan Leal, « Law Reform: An Imperative of Justice », dans Donald C. MacDonald, dir., The Government and Politics of Ontario, Scarborough, Nelson, [3" édition], 1985, p. 358-381.

${ }^{14}$ Richard Gathercole, «Humanizing the Legal System », dans Donald C. MacDonald, dir., The Government \& Politics of Ontario, Toronto, Van Nostrand Reinhold, 1980 [1975], p. 406-427.

${ }^{15}$ Roy McMurtry, « Celebrating a Quarter Century of Community Legal Clinics in Ontario », Osgoode Hall Law Journal, vol. 35, n 3 (1997), p. 425-430.

${ }^{16}$ Roy McMurtry, "The Legal Profession and Public Service", conférence prononcée lors du Third Colloquium on the Legal Profession, Toronto, The Upper Canada Law Society, octobre 2004, p. 22-23.

${ }^{17}$ Linda Cardinal, Chroniques d'une vie politique mouvementée. L'Ontario francophone de 1986 à 1996, Ottawa, Le Nordir, 2001, p. 46-52.

${ }^{18}$ Certains conseils scolaires refusent d'entériner la construction d'écoles homogènes de langue française. Des crises scolaires éclatent dès la fin des années 1960 et au début des années 1970 à Ottawa et Sturgeon Falls et, quelques années plus tard, à Elliott Lake, Kirkland Lake, Géraldton-Longlac, East York et Windsor. La plus célèbre a lieu dans la petite ville de Penetanguishene située au bord du Lac Huron. Elle retient l'attention des médias canadiens alors que le Québec est en pleine campagne référendaire. Voir Stéphane Lang, «Les luttes pour les écoles secondaires de langue française en Ontario, 19701988 ", dans La présence française en Ontario : 1610, passeport pour 2010, http:// www.crccf.uottawa.ca/passeport/IV/IVD1c/IVD1c.html, consulté le [21 avril 2007].

${ }^{19}$ Linda Cardinal, Stéphane Lang, Nathalie Plante, Anik Sauvé et Chantal Terrien, Les services.en français dans le domaine de la justice en Ontario: un état des lieux, Toronto, Ministère du Procureur général, 2005, p. 27.

${ }^{20}$ Matthew Hayday étudie les changements effectués par les gouvernements de John Robarts et de Davis à la fin des années 1960 et au début des années 1970 dans son livre Bilingual Today, United Tomorrow: Official Languages in Education and Canadian Federalism, Montréal \& Kingston, McGill-Queen's University Press, 2005, p. 50-51, 77-81.

${ }^{21}$ Sur l'origine du mouvement C'est l'temps ! et son impact sur McMurtry et le gouvernement de Davis, voir Fernand Carrière, « La métamorphose de la communauté franco-ontarienne, 1960-1985», dans Cornelius Jaenen, dir., 
Les Franco-Ontariens, Ottawa, Presses de l'Université d'Ottawa, 1994, p. 326328.

${ }^{22}$ Yves Chartier, "Mouvement C'est l'temps», s.l.n.d., UO, CRCCF, Fonds Mouvement C'est l'temps !, (P85), P85-1/3/8.

${ }^{23}$ Le mouvement C'est l'temps! rayonne principalement au niveau de la province de l'Ontario. La Fédération des francophones hors Québec (FFHQ), aujourd'hui la Fédération des communautés francophones et acadienne (FCFA), est fondée en 1975 suite à la publication de son premier manifeste, Les héritiers de Lord Durham. À l'époque, l'organisation n'est pas un interlocuteur important avec le gouvernement ontarien et ne le sera jamais véritablement, son mandat étant davantage pancanadien que provincial. Cette situation a sans doute contribué à la mobilisation des juristes francophones hors Québec au sein d'un nouveau réseau associatif, particulièrement en Ontario et au Manitoba où les francophones cherchaient à obtenir des droits scolaires et à faire reconnaitre leur province officiellement bilingue. Pour plus de détails sur le contexte de l'émergence du mouvement C'est l'temps !, voir Linda Cardinal et al., op. cit., p. 28-38. Pour le Manitoba, voir Raymond Hébert, Manitoba's French-language crisis: a cautionary tale, Montréal, McGill-Queen's University Press, 2004.

${ }^{24}$ Sur la question, voir aussi l'étude de Christiane Rabier, « Du compromis à l'antagonisme : l'axe Québec-Ottawa-Toronto et l'Ontario français, 19601982 », Francophonies d'Amérique, nº 9 (1999), p. 183-197.

${ }^{25}$ Roy McMurtry, Le bilinguisme et le droit : la nouvelle réalité : $10^{\circ}$ anniversaire de la consécration du fransais comme langue judiciaire officielle en Ontario, Toronto, Cour de Justice de l'Ontario, 1995, p. 8.

${ }^{26}$ Ramsay Cook, «Quebec's important role », Toronto Star, 6 avril 1982, p. C12.

${ }^{27}$ On peut retrouver ces textes dans le site Internet de l'Empire Club of Canada : The Empire Club Addresses,

http://www.empireclubfoundation.com/search.asp, [consulté le 6 juin 2006].

${ }^{28}$ Ernest Renan, Qu'est-ce qu'une nation ? et autres essais politiques, Paris, Presses Pocket, 1992. Roy McMurtry, « Canada after February 18, 1980 », Empire Club of Canada Addresses, vol. 77 (1979-1980), p. 284. 
${ }^{29}$ [Roy McMurtry, "Notes for an Address by the Honourable R. Roy McMurtry, Attorney General », $3^{\mathrm{e}}$ Congrès de l'AJEFO, 13 novembre 1982, p. 8-9], UO, CRCCF, Fonds AJEFO (C126), C126-2/26/4.

${ }^{30}$ Roy McMurtry, «The Search for a Constitutional Accord - A Personal Memoir ", Queen's Law Journal, vol. 51, n" 8 (1982-1983), p. 38.

${ }^{31}$ [Roy McMurtry, "Notes pour une allocution de l'honorable R. Roy McMurtry, procureur général de l'Ontario, à la Chambre de commerce du district de Montréal », Montréal (12 novembre 1980), p. 11], UO, CRCCF, Fonds ACFO (C2), C2/470/19.

${ }^{32}$ Roy McMurtry, « Canada after February 18, 1980 », p. 282-292.

${ }^{33}$ [Roy McMurtry, « Discours de l'honorable Roy R. McMurtry (Congrès de l'AJEFO) », novembre 1981], UO, CRCCF, Fonds AJEFO (C126), S25-3/ 10.

${ }^{34}$ Roy McMurtry, "The Search for a Constitutional Accord - A Personal Memoir ", Queen's Law Journal, vol. 51, nº 8 (1982-1983), p. 58.

${ }^{35}$ [Discours de Robert Paris, président de l'AJEFO, lors du congrès de l'AJEFO de 1983], UO, CRCCF, Fonds AJEFO (C126), C126-2/26/5.

${ }^{36} \mathrm{La}$ gestion scolaire pleine et entière ne sera octroyée qu'en 1997 par le premier ministre conservateur Mike Harris. Michael Behiels étudie le rôle des acteurs de la communauté franco-ontarienne dans l'évolution de la gouvemance scolaire francophone en Ontario depuis le début des années 1970 dans le troisième chapitre de son livre La francophonie canadienne : renouveau constitutionel et gouvernance scolaire, Ottawa, Presses de l'Université d'Ottawa, 2005.

${ }^{37}$ [Roy McMurtry, "Notes for an Address by the Honourable R. Roy McMurtry, Attorney General ", $3^{\circ}$ Congrès de l'AJEFO, 13 novembre 1982, p. 4], UO, CRCCF, Fonds AJEFO, C126-2/26/4.

${ }^{38}$ Ibid.

${ }^{39}$ Ibid.

47) L'Ontario soutient ainsi que : "As the dimensions of nationhood developed the formal mechanisms for amendments to the B.N.A. Act evolved as a matter between the federal government and the government of the United Kingdom. Internal Canadian political realities might well require consultation with the provinces. It was clearly an unhappy circumstance for the provinces that they did not enjoy in strict law, any legal status or powers with 
respect to the B.N.A. Act ». Roy McMurtry, « The Search for a Constitutional Accord - A Personal Memoir», p. 50.

${ }^{41}$ Ibid., p. 51.

${ }^{42}$ [Roy McMurtry, « Discours prononcé par l'honorable R. Roy McMurtry », $1^{\text {er }}$ congrès de l'AJEFO, novembre 1980, p. 53], UO, CRCCF, Fonds AJEFO (C126), C126-2/15/23.

${ }^{43}$ Ibid. Souligné dans le texte.

${ }^{44}$ Ibid., p. 54.

${ }^{45}$ Lettre d'Yves Saint-Denis, président de l'Association canadienne-française de l'Ontario, à l'honorable Roy McMurtry, procureur général de l'Ontario, Ottawa (6 novembre 1980)], UO, CRCCF, Fonds ACFO (C2), C2/470/18.

${ }^{46}$ [Lettre de Roy McMurtry à Yves Saint-Denis, président de l'ACFO (10 décembre 1980)], UO, CRCCF, Fonds ACFO (C2), C2/470/18.

${ }^{47}$ Lettre de Roy McMurtry à Robert Paris, président de l'AJEFO (30 décembre 1980)], UO, CRCCF, Fonds AJEFO (C126), C126-2/26/4.

${ }^{48}$ [Roy McMurtry, "Notes pour une allocution de l'honorable R. Roy McMurtry, procureur général de l'Ontario, à la Chambre de commerce du district de Montréal », Montréal (12 novembre 1980), p. 8], UO, CRCCF, Fonds ACFO (C2), C2/470/19.

49) Voir Fernand Harvey, "La commission Pepin-Robarts, le Québec et la francophonie canadienne », dans Jean-Pierre Wallot (dir.), Le débat qui n'apas eu lieu : La Commission Pepin-Robarts, quelques vingt ans après, Ottawa, Presses de l'Université d'Ottawa, 2002, p. 29-45 ; et Linda Cardinal et Marie-Ėve Hudon, "Les réactions des minorités francophones hors Québec au rapport de la Commission sur l'unité canadienne ", ibid., p. 47-58.

${ }^{50}$ Discours de Roy McMurtry au congrès de l'AJEFO de novembre 1981, op. cit., p. 2.

${ }^{51}$ Roy McMurtry, «The Search of a Constitutional Accord », p. 55-56 et 66. À ce jour, les historiens et les politicologues n'ont pas tenu compte du témoignage de McMurtry comme acteur impliqué directement dans cet événement grandement symbolique que représente le rapatriement de la Constitution et l'adoption de la Charte. Ils ont négligé son rôle dans l'interprétation des intentions des responsables du rapatriement et sa compréhension du sentiment de trahison ressenti par le gouvernement québécois à l'époque.

${ }^{52}$ Ibid., p. 68. 
${ }^{53}$ Discours de Roy McMurtry au congrès de l'AJEFO de novembre 1981, p. 7. ${ }^{54}$ [Roy McMurtry, "Notes for an Address by the Honourable R. Roy McMurtry, Attorney General », $3^{\mathrm{e}}$ Congrès de l'AJEFO, 13 novembre 1982, $\mathrm{p}$. 8-9], UO, CRCCF, Fonds AJEFO (C126), C126-2/26/4.

${ }^{55}$ Maurice Jannard, « Accord du lac Meech : Roy McMurtry soutient que la clause de la société distincte a peu de portée », La Presse, 5 décembre 1989, p. B1.

${ }^{56}$ Bronwyn Kienapple, « Chief Justice instrumental in creation of Charter of Rights and Freedom », The Varsity, 17 mars 2003.

${ }^{57}$ [Copie de la lettre de Roy McMurtry à Colin Vezina, éditeur du North Bay Nugget, 10 janvier 1983], UO, CRCCF, Fonds AJEFO (C126), C126-2/22/9.

${ }^{58}$ Roy McMurtry, Le bilinguisme et le droit : la nouvelle réalité : $10^{\circ}$ anniversaire de la consécration du français comme langue judiciaire officielle en Ontario, Toronto, Cour de Justice de l'Ontario, 1995, p. 7. Dans son rappel historique, McMurtry indique clairement que seul l'appui inconditionnel du premier ministre Davis lui a permis de faire accepter son projet de bilinguisme judiciaire.

${ }^{59}$ [Memorandum intitulé « French-language services » de l'honorable R. Roy McMurtry, procureur général, à tous les hauts fonctionnaires des différentes divisions administratives de son ministère, 23 juin 1980], UO, CRCCF, Fonds ACFO (C2), C2/470/18.

${ }^{61}$ Discours de Roy McMurtry au congrès de l'AJEFO de novembre 1980, p. 10.

${ }^{61}$ Commission de l'Unité canadienne, Se retrouver: observations et recommandations, Hull, Ministère des Approvisionnements et Service Canada, 1979. 